\title{
STABILITY OF CAPILLARY HYPERSURFACES IN A EUCLIDEAN BALL
}

\author{
HAIZHONG LI AND CHANGWEI XIONG
}

\begin{abstract}
We study the stability of capillary hypersurfaces in a unit Euclidean ball. It is proved that if the mass center of the generalized body enclosed by the immersed capillary hypersurface and the wetted part of the sphere is located at the origin, then the hypersurface is unstable. An immediate result is that all known examples except the totally geodesic ones and spherical caps are unstable.
\end{abstract}

\section{INTRODUCTION}

Capillarity is an important physical phenomena, which occurs when two different materials contact and do not mix. Given a container $B$ with an incompressible liquid drop $T$ in it, the interface of the liquid and the air is a capillary surface $M$. In absence of gravity, the interface $M$ is of constant mean curvature and the contact angle of $M$ to the boundary $\partial B$ is constant. One should compare this setting with soap bubble (resp. soap film), where the surface has no boundary (resp. fixed boundary) and constant mean curvature.

The literature for the study of capillarity is extensive and we refer to the book of Finn [5], where the treatment of the theory is mainly in the nonparametric case and in the more general situation of presence of gravity. Also we mention [6] for a more recent survey about this topic.

In this paper we are concerned with the special case that the container $B$ is a unit Euclidean ball and no gravity is involved. We study the (weak) stability for capillary hypersurfaces. This problem has been discussed by Ros and Souam 13, where they dealt with surface case and obtained some topological and geometrical restrictions. For the hypersurface case with free boundary (the contact angle is $\pi / 2$ ), Ros and Vergasta also proved some interesting results in 14 .

Applying the same argument as in the proof of Proposition 1.1 in [13], we know that the hyperplanes and the spherical caps in a unit Euclidean ball are capillarily stable. Recently, Marinov 12 proved that, for surface case, all other known examples are unstable. We generalize Marinov's result to the hypersurface case. In fact we prove the following theorem.

2010 Mathematics Subject Classification. 53A10, 49Q10.

Key words and phrases. capillary hypersurface; instability; spherical boundary.

The research of the authors was supported by NSFC No. 11271214. 
Theorem 1. Let $x: M^{n} \rightarrow \mathbb{R}^{n+1}$ be an immersed capillary hypersurface in a unit Euclidean ball $B^{n+1}$ and $\Omega$ the wetted part of the boundary of the ball. Denote by $T$ the generalized body enclosed by $x(M)$ and $\Omega$. If the mass center of $T$ is at the origin, the capillary hypersurface $M$ is unstable.

Here since we assume $M$ is immersed, $x(M)$ may have self-intersections. Thus we need to consider the generalized body $T$. When $M$ is embedded, $T$ is understood in the common sense. See Remark 1 below for more explanation.

For $n=2$, our Theorem 1 reduces to Marinov's result in 12 . We also note that his argument relies on the conformal coordinates, which can not be generalized to the higher dimensional case.

Applying Theorem 11 to Delaunay hypersurfaces, we get the following Corollary 2, Recall that Delaunay hypersurfaces are the ones of revolution with constant mean curvature. By Proposition 4.3 in [11], Delaunay hypersurfaces are classified as: an unduloid, cylinder, nodoid, sphere, catenoid, or a hyperplane. To guarantee the portion of a Delaunay hypersurface in a Euclidean ball is also capillary, it shall have some symmetry. See Section 2 below for more details. And in that case, we call it Delaunay capillary hypersurface. From Theorem 1 we have

Corollary 2. The only stable Delaunay capillary hypersurface $M^{n}$ in a unit Euclidean ball $B^{n+1}$ is a totally geodesic hyperplane or a spherical cap.

Our approach for proving Theorem 1 is as follows. In higher dimensional case, we find that we can construct a conformal killing vector field $Y[\xi]$ for any fixed $\xi \in \mathbb{S}^{n}$ from the natural conformal transformation family on $B^{n+1}$. Using the normal part $\langle Y[\xi], N\rangle$ as the test function we can define a symmetric quadratic form $Q\left(\xi_{1}, \xi_{2}\right)$. By summing $Q$ over $(n+1)$ coordinate directions we find $Q$ has at least one negative eigenvalue. This summation technique can be compared with J. Simons' work [16]. At last, under the hypothesis of Theorem 1 we can derive the instability of the hypersurface. Our argument indicates that this conformal field is very important and we can use it to conclude that the mass center of minimal submanifolds with free boundary in a unit Euclidean ball is at the origin (See Proposition 11). We refer the readers to $7 \cdot 9]$ for the very recent work on the minimal submanifolds with free boundary.

At last, as an application of our argument, we give a new proof of the classical result due to Barbosa and do Carmo [1], which states that the only closed stable immersed hypersurface of constant mean curvature in $\mathbb{R}^{n+1}$ is the round sphere.

An outline of this paper is as follows. In Section 2 after fixing some notations and definitions, we prove the stability of hyperplanes and spherical caps. Then we construct the crucial conformal vector field. We also review some known facts about the Delaunay hypersurfaces. In Section 3 we give the proof of Theorem 1. In last section, we discuss some applications of our method. 


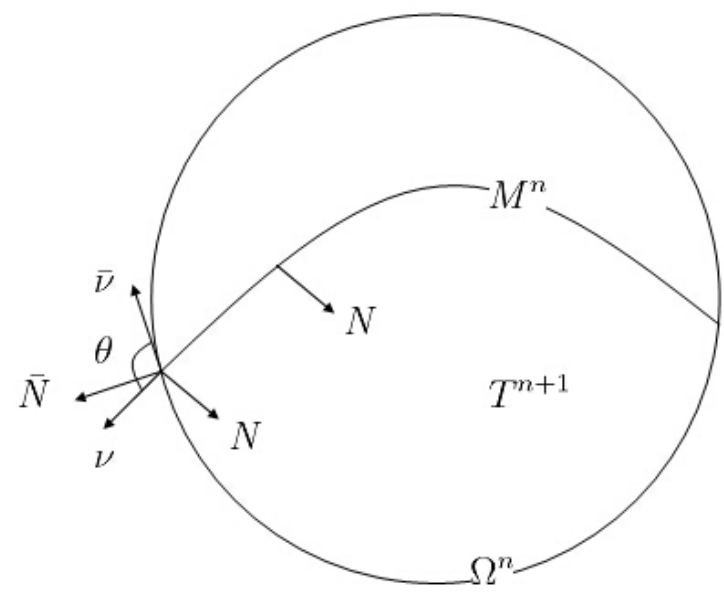

Figure 1. A typical illustration

\section{Preliminaries}

2.1. Notations and definitions. Let $x: M^{n} \rightarrow \mathbb{R}^{n+1}$ be an orientable immersed hypersurface in the unit Euclidean ball $B^{n+1} \subset \mathbb{R}^{n+1}$ with $x($ int $M) \subset$ $B^{n+1}$ and $x(\partial M) \subset \partial B^{n+1}$. Suppose $\Omega \subset \partial B^{n+1}$ such that $\partial \Omega=x(\partial M)$. And denote by $T \subset B^{n+1}$ the part of ball satisfying $\partial T=x(M) \cup \Omega$.

Remark 1. If $x(M)$ has self-intersections, $T$ may be viewed as the finite union of some domains $T_{i}, i=1, \cdots, m$, i.e. $T=\cup_{i=1}^{m} T_{i}$. Here $T_{i}$ may intersect with each other. If there are not one choices for $\left\{T_{i}\right\}_{i=1}^{m}$, choose one and fix it. In the proof we will see that only the property $\partial T=x(M) \cup \Omega$ is needed. And if there is no confusion, we will write $M$ (resp. $\partial M$ ) for $x(M)$ (resp. $x(\partial M))$ for simplicity.

Let $N$ be the unit normal of $M$ pointing inwards to $T$ and $\bar{N}$ the unit outward normal of $\partial B^{n+1}$. Denote by $\nu$ and $\bar{\nu}$ the conormals of $\partial M$ in $M$ and $\Omega$, respectively. Let $D$ (resp. $\nabla$ ) be the connection of $\mathbb{R}^{n+1}$ (resp. $M$ ). Then the second fundamental form of $M$ in $\mathbb{R}^{n+1}$ is given by $\sigma\left(X_{1}, X_{2}\right)=$ $\left\langle D_{X_{1}} X_{2}, N\right\rangle$ for $\forall X_{1}, X_{2} \in T M$. When taking an orthonormal basis $\left\{e_{i}\right\}_{i=1}^{n}$ on $T M$, we also denote by $h_{i j}$ the components $\sigma\left(e_{i}, e_{j}\right)$. So the mean curvature $H$ of $M$ is $H=\frac{1}{n} \sum_{i=1}^{n} h_{i i}$. And the second fundamental form of $\partial B$ in $\mathbb{R}^{n+1}$ is given by $\Pi\left(Y_{1}, Y_{2}\right)=\left\langle D_{Y_{1}} Y_{2},-\bar{N}\right\rangle$ for $\forall Y_{1}, Y_{2} \in T(\partial B)$. At last let $\theta \in(0, \pi)$ be the angle between $\nu$ and $\bar{\nu}$. See Figure 1 for an illustration.

Following [13, we discuss the variation of $M$.

Definition 2.1. An admissible variation of $x: M^{n} \rightarrow \mathbb{R}^{n+1}$ is a differentiable map $X:(-\varepsilon, \varepsilon) \times M \rightarrow \mathbb{R}^{n+1}$ so that $X_{t}: M^{n} \rightarrow \mathbb{R}^{n+1}, t \in(-\varepsilon, \varepsilon)$ 
given by $X_{t}(p)=X(t, p), p \in M$ is an immersion satisfying $X_{t}($ int $M) \subset$ int $B$ and $X_{t}(\partial M) \subset \partial B$ for all $t$, and $X_{0}=x$.

Now for given $\theta \in(0, \pi)$, we define a energy functional

$$
E(t)=|M(t)|-\cos \theta|\Omega(t)|
$$

where $|\cdot|$ denotes the area function. And the volume functional can be defined as

$$
V(t)=\int_{[0, t] \times M} X^{*} d v
$$

where $d v$ is the standard volume element of $\mathbb{R}^{n+1}$.

Under these constraints, we have

Definition 2.2. An immersed hypersurface $x: M^{n} \rightarrow \mathbb{R}^{n+1}$ is called capillary if $E^{\prime}(0)=0$ for any admissible volume-preserving variation of $x$.

Note that we have the following formulas,

$$
\begin{aligned}
E^{\prime}(0) & =-n \int_{M} H f d a+\int_{\partial M}\langle Y, \nu-\cos \theta \bar{\nu}\rangle d s, \\
V^{\prime}(0) & =-\int_{M} f d a
\end{aligned}
$$

where $Y$ is the variational vector field $Y(p)=\left.\frac{\partial X}{\partial t}(p)\right|_{t=0}, f$ its normal component $f=\langle Y, N\rangle$, and $d a$ and $d s$ are the corresponding area elements.

From these formulas we see that $M$ is capillary if and only if it has constant mean curvature and makes constant contact angle $\theta$ with $\partial B$. Furthermore, one can compute the second derivative at $t=0$ with respect to an admissible volume-preserving variation to get (see e.g. the appendix of [13])

$$
E^{\prime \prime}(0)=-\int_{M}\left(\Delta f+\left(|\sigma|^{2}+R i c(N)\right) f\right) f d a+\int_{\partial M}\left(\frac{\partial f}{\partial \nu}-q f\right) f d s,
$$

where $f \in \mathcal{F}:=\left\{f \in H^{1}(M), \int_{M} f d a=0\right\}, \operatorname{Ric}(N)$ is the Ricci curvature of the ambient space and

$$
q=\frac{1}{\sin \theta} \Pi(\bar{\nu}, \bar{\nu})+\cot \theta \sigma(\nu, \nu) .
$$

In our setting, $\operatorname{Ric}(N)=0$ and $\Pi(\bar{\nu}, \bar{\nu})=1$.

Definition 2.3. A capillary hypersurface $M$ is called (weakly) stable if $E^{\prime \prime}(0) \geq 0$ for all $f \in \mathcal{F}$.

In the sequel we will denote by $\partial^{2} E(\phi)$ the quantity $E^{\prime \prime}(0)$ for a given function $\phi$. 
2.2. Stable examples of capillary hypersurfaces. First we prove the stability of totally geodesic capillary hypersurfaces and spherical caps. The proof is similar to that of Proposition 1.1 in [13]. We include it for completeness.

Proposition 3. Let $B^{n+1} \subset \mathbb{R}^{n+1}$ be a unit Euclidean ball. Then totally geodesic capillary hypersurfaces and spherical caps are stable.

Proof. First assume $M$ is a totally geodesic capillary hypersurface, i.e., an $n$-dimensional ball $B^{n}(R)$ with radius $R$ in $B^{n+1}$. Then the contact angle $\theta$ satisfies $\sin \theta=R$. By the definition of stability, we have to prove

$$
\int_{M}|\nabla f|^{2} d a \geq \frac{1}{R} \int_{\partial M} f^{2} d s, \text { for } \forall f \in \mathcal{F} .
$$

Consider now the $(n+1)$-dimensional ball $B^{\prime}$ of radius $R$ having $M$ as an equatorial totally geodesic hypersurface. Then by [2], $M$ is area minimizing for partitioning problem in $B^{\prime}$. Thus $M$ is stable in $B^{\prime}$, which is equivalent to the inequality (6).

Next assume $M$ is a spherical cap in $B^{n+1}$ with $R$ the radius of the sphere containing $M$ and $\theta$ the contact angle. Consider the $n$-dimensional hyperplane $P$ containing $\partial M$. Then $M$ is a capillary hypersurface in a halfspace with a contact angle $\theta^{\prime}$. By [10], $M$ is stable in the halfspace, which means

$$
\int_{M}\left(|\nabla f|^{2}-\frac{n}{R^{2}} f^{2}\right) d a \geq \frac{\cot \theta^{\prime}}{R} \int_{\partial M} f^{2} d s, \text { for } \forall f \in \mathcal{F} .
$$

Elementary calculation leads to

$$
\frac{1}{\sin \theta}+\frac{\cot \theta}{R}=\frac{\cot \theta^{\prime}}{R}
$$

Now (7) and (8) together yield the stability of $M$ in $B^{n+1}$.

2.3. Conformal transformations on the Euclidean ball. Now we construct a conformal vector field. Fix a vector $a \in B^{n+1}$. Then (see e.g. Section 3.8 in [15])

$$
\varphi_{a}(x)=\frac{\left(1-|a|^{2}\right) x-\left(1-2\langle a, x\rangle+|x|^{2}\right) a}{1-2\langle a, x\rangle+|a|^{2}|x|^{2}}
$$

defines a map from $B^{n+1}$ to $B^{n+1}$ and from $\mathbb{S}^{n}$ to $\mathbb{S}^{n}$, since we have

$$
1-\left|\varphi_{a}(x)\right|^{2}=\frac{\left(1-|a|^{2}\right)\left(1-|x|^{2}\right)}{1-2\langle a, x\rangle+|a|^{2}|x|^{2}} .
$$

Moreover $\varphi_{a}$ is conformal. In fact, by a direct calculation we can check that

$$
\left|d \varphi_{a}\right|^{2}=\left(\frac{1-|a|^{2}}{1-2\langle a, x\rangle+|a|^{2}|x|^{2}}\right)^{2}|d x|^{2} .
$$


Note that $\varphi_{a}(a)=0, \varphi_{a}(0)=-a, \varphi_{a}$ fixes two points $\pm \frac{a}{|a|}$ and $\varphi_{0}$ is an identity.

Next fix $\xi \in \mathbb{S}^{n}$. Let $a=t \xi$ with $-1<t<1$. Then

$$
f_{t}(x)=\varphi_{t \xi}(x)=\frac{\left(1-t^{2}\right) x-\left(1-2 t\langle\xi, x\rangle+|x|^{2}\right) t \xi}{1-2 t\langle\xi, x\rangle+t^{2}|x|^{2}}
$$

is a family of conformal transformations with parameter $t$.

Thus $f_{t}$ determines a conformal vector field $Y[\xi]$ as follows.

$$
Y[\xi]=\left.\frac{d}{d t}\right|_{t=0} f_{t}(x)=-\left(1+|x|^{2}\right) \xi+2\langle\xi, x\rangle x .
$$

Note that $Y[\xi]$ is tangential along the sphere $\mathbb{S}^{n}$, since for $\forall x \in \mathbb{S}^{n}$,

$$
\langle Y[\xi], x\rangle=-\left(1+|x|^{2}\right)\langle\xi, x\rangle+2\langle\xi, x\rangle|x|^{2}=0 .
$$

2.4. Delaunay hypersurfaces in Euclidean space. In this subsection, following [11] we review some facts about Delaunay hypersurfaces, which is rotational and of constant mean curvature $H$. These hypersurfaces are the models we are concerned with in Theorem 1 .

Let $M^{n} \subset \mathbb{R}^{n+1}$ be a hypersurface which is invariant under the action of the orthogonal group $O(n)$ fixing the $x^{1}$-axis. Assume $M$ is generated by a curve $\Gamma$ contained in the $x^{1} x^{2}$-plane. Then it suffices to determine the curve $\Gamma$.

Parametrize the curve $\Gamma=\left(x^{1}, x^{2}\right)$ by arc-length $s$. Denote by $\alpha$ the angle between the tangent to $\Gamma$ and the positive $x^{1}$-direction and choose the normal vector $N=(\sin \alpha,-\cos \alpha)$. Then $\left(x^{1}, x^{2} ; \alpha\right)$ satisfies the following system of ordinary differential equations

$$
\left\{\begin{array}{l}
\left(x^{1}\right)^{\prime}=\cos \alpha \\
\left(x^{2}\right)^{\prime}=\sin \alpha \\
\alpha^{\prime}=-n H+(n-1) \frac{\cos \alpha}{x^{2}} .
\end{array}\right.
$$

The first integral of this system is given by

$$
\left(x^{2}\right)^{n-1} \cos \alpha-H\left(x^{2}\right)^{n}=F,
$$

where the constant $F$ is called the force of the curve $\Gamma$ and it together with $H$ will determine the curve as follows. (See Proposition 4.3 in [11])

Proposition 4. The curve $\Gamma$ and the hypersurface $M$ generated by $\Gamma$ have the following several possible types.

(1) If $F H>0$ then $\Gamma$ is a periodic graph over the $x^{1}$-axis. It generates a periodic embedded unduloid, or a cylinder.

(2) If $F H<0$ then $\Gamma$ is a locally convex curve and $M$ is a nodoid, which has self-intersections.

(3) If $F=0$ and $H \neq 0$ then $M$ is a sphere.

(4) If $H=0$ and $F \neq 0$ we obtain a catenary which generates an embedded catenoid $M$ with $F>0$ if the normal points down and $F<0$ if the normal points up. 
(5) If $H=0$ and $F=0$ then $\Gamma$ is a straight line orthogonal to the $x^{1}$-axis which generates a hyperplane.

(6) If $M$ touches the $x^{1}$-axis, then it must be a sphere or a hyperplane.

(7) The curve $\Gamma$ is determined, up to translation along the $x^{1}$-axis, by the pair $(H, F)$.

From this proposition, it is easy to see if $M^{n}$ is the portion of an unduloid, cylinder, nodoid or a catenoid in a unit Euclidean ball $B^{n+1}$ with revolution axis $x^{1}$ and moreover $M$ is symmetric with respect to the hyperplane $\left\{x^{1}=0\right\}$, then $M$ is a capillary hypersurface in $B^{n+1}$. In that case we call them Delaunay capillary hypersurfaces in $B^{n+1}$. Furthermore the generalized body $T$ enclosed by $M$ and the wetted part of the sphere has the mass center at the origin. So Theorem 1 is applicable.

\section{INSTABILITY OF CAPILLARY HYPERSURFACES}

With the preparations above, we can define a "test function"

$$
\phi[\xi]=\langle Y[\xi], N\rangle=\left\langle-\left(1+|x|^{2}\right) \xi+2\langle\xi, x\rangle x, N\right\rangle .
$$

We mention that we will also use the following expression of $\phi[\xi]$

$$
\phi[\xi]=\left\langle\xi,-\left(1+|x|^{2}\right) N+2\langle x, N\rangle x\right\rangle .
$$

Recall the second variational formula

$$
\partial^{2} E(\phi)=-\int_{M} L \phi \cdot \phi d a+\int_{\partial M}\left(\phi_{\nu}-q \phi\right) \phi d s,
$$

where $L=\Delta+|\sigma|^{2}$ and $q=\frac{1}{\sin \theta}+\cot \theta \sigma(\nu, \nu)$.

Now we can prove the following lemmas.

Lemma 5. $\nu$ is a principal direction for $\sigma$ along $\partial M$. In particular, $D_{\nu} N=$ $-\sigma(\nu, \nu) \nu$.

Proof. It suffices to prove that, for $\forall X \in T_{p}(\partial M), \sigma(\nu, X)=0$. In fact, we have

$$
\begin{aligned}
\sigma(\nu, X) & =\left\langle D_{X} \nu, N\right\rangle \\
& =\left\langle D_{X}(\cos \theta \bar{\nu}+\sin \theta \bar{N}),-\sin \theta \bar{\nu}+\cos \theta \bar{N}\right\rangle \\
& =\left\langle D_{X} \bar{\nu}, \bar{N}\right\rangle \\
& =-I I(\bar{\nu}, X) \\
& =0,
\end{aligned}
$$

where we used $\theta$ is constant, $\bar{\nu}$ and $\bar{N}$ are unit vectors, and $\partial B$ is totally umbilical. Thus we complete the proof of Lemma 5.

Lemma 6. Along $\partial M$, there holds

$$
\phi_{\nu}-q \phi=0 .
$$


Proof. First from (13) and Lemma 5 we have

$$
\begin{aligned}
\phi_{\nu} & =\left\langle\xi,-\left(1+|x|^{2}\right) N+2\langle x, N\rangle x\right\rangle_{\nu} \\
& =\left\langle\xi,-2\langle x, \nu\rangle N+\left(1+|x|^{2}\right) \sigma(\nu, \nu) \nu-2\langle x, \sigma(\nu, \nu) \nu\rangle x+2\langle x, N\rangle \nu\right\rangle \\
& =2\langle\xi,-\langle x, \nu\rangle N+\sigma(\nu, \nu)(\nu-\langle x, \nu\rangle x)+\langle x, N\rangle \nu\rangle,
\end{aligned}
$$

where in the third line we used $|x|=1$ along $\partial M$.

Next noticing that $x=\bar{N}=\cos \theta N+\sin \theta \nu$, we get

$$
\begin{aligned}
\phi_{\nu} & =2\langle\xi,-\sin \theta N+\sigma(\nu, \nu)(\nu-\sin \theta(\cos \theta N+\sin \theta \nu))+\cos \theta \nu\rangle \\
& =2\langle\xi,(\sigma(\nu, \nu) \cos \theta+1)(\cos \theta \nu-\sin \theta N)\rangle .
\end{aligned}
$$

On the other hand, there holds

$$
\begin{aligned}
q \phi & =\left(\frac{1}{\sin \theta}+\cot \theta \sigma(\nu, \nu)\right)\left\langle\xi,-\left(1+|x|^{2}\right) N+2\langle x, N\rangle x\right\rangle \\
& =\left(\frac{1}{\sin \theta}+\cot \theta \sigma(\nu, \nu)\right) 2\langle\xi,-N+\cos \theta(\cos \theta N+\sin \theta \nu)\rangle \\
& =\left(\frac{1}{\sin \theta}+\cot \theta \sigma(\nu, \nu)\right) 2\left\langle\xi,-\sin ^{2} \theta N+\cos \theta \sin \theta \nu\right\rangle \\
& =(1+\cos \theta \sigma(\nu, \nu)) 2\langle\xi,-\sin \theta N+\cos \theta \nu\rangle,
\end{aligned}
$$

where again in the second line we used $|x|=1$ along $\partial M$.

Hence we obtain

$$
\phi_{\nu}-q \phi=0
$$

The next lemma, which indicates the geometric meaning of Lemma 6 , may have its own interest. Thus we also include it here.

Lemma 7. Under the flow $f_{t}$, there holds

$$
\left.\frac{d}{d t}\right|_{t=0} \theta(t)=\phi_{\nu}-q \phi
$$

In particular, since $f_{t}$ is conformal (angle preserving), $\phi_{\nu}-q \phi=0$.

Proof. Following [13, we denote by a "prime" the convariant derivative $\left.\frac{D}{d t}\right|_{t=0}$. Also by the appendix of $[13]$, we have

$$
\begin{aligned}
& \nu^{\prime}=\left(\frac{\partial \phi}{\partial \nu}+\sigma\left(Y_{0}, \nu\right)\right) N+\phi S_{0}(\nu)-\phi \sigma(\nu, \nu) \nu-S_{1}\left(Y_{1}\right)+\cot \theta \tilde{\nabla} \phi, \\
& \bar{\nu}^{\prime}=-\Pi(Y, \bar{\nu}) \bar{N}-S_{2}\left(Y_{1}\right)+\frac{1}{\sin \theta} \tilde{\nabla} \phi,
\end{aligned}
$$

where $\tilde{\nabla}$ denotes the gradient on $\partial M, Y_{0}$ (resp. $\left.Y_{1}\right)$ the tangent part of the variational vector field $Y$ of $M$ (resp. to $\partial M), S_{0}$ the shape operator of $M$ in $\mathbb{R}^{n+1}$ with respect to $N$, and $S_{1}$ (resp. $S_{2}$ ) the shape operator of $\partial M$ in $M$ (resp. $\partial B$ ) with respect to $\nu$ (resp. $\bar{\nu}$ ). 
Note that $\cos \theta(t)=\langle\nu, \bar{\nu}\rangle$, which implies

$$
-\left.\sin \theta \frac{d}{d t}\right|_{t=0} \theta(t)=\left\langle\nu^{\prime}, \bar{\nu}\right\rangle+\left\langle\nu, \bar{\nu}^{\prime}\right\rangle .
$$

Taking into account that

$$
\begin{aligned}
\bar{\nu} & =-\sin \theta N+\cos \theta \nu \\
\bar{N} & =\cos \theta N+\sin \theta \nu
\end{aligned}
$$

we have

$$
\begin{aligned}
-\left.\sin \theta \frac{d}{d t}\right|_{t=0} \theta(t) & =\left\langle\left(\frac{\partial \phi}{\partial \nu}+\sigma\left(Y_{0}, \nu\right)\right) N+\phi S_{0}(\nu)-\phi \sigma(\nu, \nu) \nu,-\sin \theta N+\cos \theta \nu\right\rangle \\
& +\langle\nu,-\Pi(Y, \bar{\nu})(\cos \theta N+\sin \theta \nu)\rangle \\
& =-\sin \theta\left(\frac{\partial \phi}{\partial \nu}+\sigma\left(Y_{0}, \nu\right)\right)-\sin \theta \Pi(Y, \bar{\nu}),
\end{aligned}
$$

or

$$
\left.\frac{d}{d t}\right|_{t=0} \theta(t)=\frac{\partial \phi}{\partial \nu}+\sigma\left(Y_{0}, \nu\right)+\Pi(Y, \bar{\nu})
$$

Again from the appendix of [13], there hold

$$
\begin{gathered}
Y_{0}=Y_{1}-\cot \theta \phi \nu, \\
Y=Y_{1}-\frac{1}{\sin \theta} \phi \bar{\nu}, \\
\sigma\left(Y_{1}, \nu\right)+\Pi\left(Y_{1}, \bar{\nu}\right)=0 .
\end{gathered}
$$

Now plugging these equalities into (17), the lemma follows immediately.

\section{Lemma 8.}

$$
L \phi=-2 n\langle\xi, N+H x\rangle .
$$

Proof. The proof is a direct calculation using moving frame method. This method is very powerful in differential geometry. Take an orthonormal basis $\left\{e_{i}, i=1, \cdots, n ; e_{n+1}=N\right\}$. Then we have the structure equations: (see e.g. [4])

$$
\begin{gathered}
d x=\sum_{i=1}^{n} \omega_{i} e_{i}, \\
d e_{i}=\sum_{j=1}^{n} \omega_{i j} e_{j}+\sum_{j=1}^{n} h_{i j} \omega_{j} e_{n+1}, \\
d e_{n+1}=-\sum_{i, j=1}^{n} h_{i j} \omega_{i} e_{j}
\end{gathered}
$$


where $\omega_{i}$ is the dual forms and $\omega_{i j}$ the connection forms. Thus there holds

$$
\begin{aligned}
\Delta \phi & =\Delta\left\langle\xi,-\left(1+|x|^{2}\right) N+2\langle x, N\rangle x\right\rangle \\
& =\left\langle\xi,-\Delta\left(\left(1+|x|^{2}\right) N\right)+2 \Delta(\langle x, N\rangle x)\right\rangle \\
& =\left\langle\xi,-\left(\Delta|x|^{2} \cdot N+2 \sum_{i=1}^{n}\left(|x|^{2}\right)_{, i} N_{, i}+\left(1+|x|^{2}\right) \Delta N\right)\right. \\
& \left.+2\left(\Delta\langle x, N\rangle \cdot x+2 \sum_{i=1}^{n}\langle x, N\rangle_{, i} x_{, i}+\langle x, N\rangle \Delta x\right)\right\rangle .
\end{aligned}
$$

Note that

$$
\begin{aligned}
\Delta|x|^{2} & =2\langle x, \Delta x\rangle+2|\nabla x|^{2} \\
& =2 n H\langle x, N\rangle+2 n, \\
\sum_{i=1}^{n}\left(|x|^{2}\right)_{, i} N_{, i} & =-2 \sum_{i, j=1}^{n}\left\langle x, e_{i}\right\rangle h_{i j} e_{j} .
\end{aligned}
$$

And using Codazzi equation $\sum_{i=1}^{n} h_{i j, i}=\sum_{i=1}^{n} h_{i i, j}=n H_{, j}=0$ we have

$$
\begin{aligned}
\Delta N & =\sum_{i=1}^{n} N_{, i i}=\sum_{i, j=1}^{n}\left(-h_{i j} e_{j}\right)_{, i} \\
& =-\sum_{i, j=1}^{n} h_{i j} h_{i j} N=-|\sigma|^{2} N .
\end{aligned}
$$

Moreover we can get

$$
\begin{aligned}
\Delta\langle x, N\rangle & =\langle\Delta x, N\rangle+2 \sum_{i=1}^{n}\left\langle x_{, i}, N_{, i}\right\rangle+\langle x, \Delta N\rangle \\
& =\langle n H N, N\rangle+2 \sum_{i, j=1}^{n}\left\langle e_{i},-h_{i j} e_{j}\right\rangle+\left\langle x,-|\sigma|^{2} N\right\rangle \\
& =-n H-|\sigma|^{2}\langle x, N\rangle, \\
\sum_{i=1}^{n}\langle x, N\rangle_{, i} x_{, i} & =\sum_{i, j=1}^{n}\left\langle x,-h_{i j} e_{j}\right\rangle e_{i}=\sum_{i, j=1}^{n}-h_{i j}\left\langle x, e_{j}\right\rangle e_{i} .
\end{aligned}
$$


Now substituting all these terms into 19 gives rise to

$$
\begin{aligned}
\Delta \phi & =\left\langle\xi,-\left((2 n H\langle x, N\rangle+2 n) \cdot N-4 \sum_{i, j=1}^{n}\left\langle x, e_{i}\right\rangle h_{i j} e_{j}-\left(1+|x|^{2}\right)|\sigma|^{2} N\right)\right. \\
& \left.+2\left(\left(-n H-|\sigma|^{2}\langle x, N\rangle\right) \cdot x-2 \sum_{i, j=1}^{n} h_{i j}\left\langle x, e_{j}\right\rangle e_{i}+\langle x, N\rangle n H N\right)\right\rangle \\
& =\left\langle\xi,\left(-2 n+\left(1+|x|^{2}\right)|\sigma|^{2}\right) N-2\left(n H+|\sigma|^{2}\langle x, N\rangle\right) x\right\rangle \\
& =\langle\xi,-2 n(N+H x)\rangle-|\sigma|^{2} \phi .
\end{aligned}
$$

Therefore,

$$
L \phi=\Delta \phi+|\sigma|^{2} \phi=-2 n\langle\xi, N+H x\rangle .
$$

Thus we obtain

$$
\partial^{2} E(\phi)=-2 n \int_{M}\langle\xi, N+H x\rangle \cdot\left\langle\xi,\left(1+|x|^{2}\right) N-2\langle x, N\rangle x\right\rangle d a .
$$

To analyze $\partial^{2} E(\phi)$, we define a quadratic form

$$
Q\left(\xi_{1}, \xi_{2}\right)=-2 n \int_{M}\left\langle\xi_{1}, N+H x\right\rangle \cdot\left\langle\xi_{2},\left(1+|x|^{2}\right) N-2\langle x, N\rangle x\right\rangle d a,
$$

for $\forall \xi_{1}, \xi_{2} \in \mathbb{S}^{n}$. Denote by $\left\{\partial_{A}\right\}_{A=1}^{n+1}$ the standard coordinate vectors in $\mathbb{R}^{n+1}$. Then we have the following lemma.

Lemma 9. $Q$ has the following properties.

(1) $Q$ is symmetric.

(2) $\operatorname{tr} Q=\sum_{A=1}^{n+1} Q\left(\partial_{A}, \partial_{A}\right) \leq 0$ with equality if and only if $|x|=$ const on $M$.

Proof. (1) First we prove $Q$ is symmetric. Note that in fact $Q$ is defined as

$$
Q\left(\xi_{1}, \xi_{2}\right)=-\int_{M} L\left(\phi\left[\xi_{1}\right]\right) \cdot \phi\left[\xi_{2}\right] d a .
$$

Then Green's formula implies

$$
Q\left(\xi_{1}, \xi_{2}\right)=-\int_{M} \phi\left[\xi_{1}\right] \cdot L\left(\phi\left[\xi_{2}\right]\right) d a+\int_{\partial M}\left(\phi\left[\xi_{1}\right]\left(\phi\left[\xi_{2}\right]\right)_{\nu}-\left(\phi\left[\xi_{1}\right]\right)_{\nu} \phi\left[\xi_{2}\right]\right) d s .
$$

But Lemma 6 yields $\left(\phi\left[\xi_{i}\right]\right)_{\nu}=q \phi\left[\xi_{i}\right], i=1,2$. So the boundary term vanishes and then

$$
Q\left(\xi_{1}, \xi_{2}\right)=Q\left(\xi_{2}, \xi_{1}\right)
$$


(2) Next we calculate $\operatorname{tr} Q$.

$$
\begin{aligned}
\operatorname{tr} Q & =\sum_{A=1}^{n+1} Q\left(\partial_{A}, \partial_{A}\right) \\
& =-2 n \int_{M} \sum_{A=1}^{n+1}\left\langle\partial_{A}, N+H x\right\rangle \cdot\left\langle\partial_{A},\left(1+|x|^{2}\right) N-2\langle x, N\rangle x\right\rangle d a \\
& =-2 n \int_{M}\left\langle N+H x,\left(1+|x|^{2}\right) N-2\langle x, N\rangle x\right\rangle d a \\
& =-2 n \int_{M}\left(H\langle x, N\rangle\left(1-|x|^{2}\right)+1+|x|^{2}-2\langle x, N\rangle^{2}\right) d a \\
& \leq-2 n \int_{M}(H\langle x, N\rangle+1)\left(1-|x|^{2}\right) d a .
\end{aligned}
$$

Also we have $\Delta|x|^{2}=2 n(H\langle x, N\rangle+1)$. Consequently,

$$
\begin{aligned}
\operatorname{tr} Q & \leq-\int_{M} \Delta|x|^{2} \cdot\left(1-|x|^{2}\right) d a \\
& =\int_{M} \nabla|x|^{2} \cdot \nabla\left(1-|x|^{2}\right) d a-\int_{\partial M} \frac{\partial|x|^{2}}{\partial \nu}\left(1-|x|^{2}\right) d s \\
& =-\int_{M}\left|\nabla\left(|x|^{2}\right)\right|^{2} d a \\
& \leq 0,
\end{aligned}
$$

where we have used $|x|=1$ on $\partial M$ to remove the boundary term. And it is easy to see $\operatorname{tr} Q=0$ if and only if $|x|=$ const.

So we complete the proof.

Thus $Q$ has at least one negative eigenvalue. But on the other hand,

$$
\operatorname{div}_{\mathbb{R}^{n+1}} Y[\xi]=\sum_{A=1}^{n+1}\left\langle D_{\partial_{A}} Y[\xi], \partial_{A}\right\rangle=2(n+1)\langle\xi, x\rangle,
$$

which by integration implies

$$
\begin{aligned}
\int_{M} \phi d a & =\int_{M}\langle Y[\xi], N\rangle d a \\
& =-\int_{T} d i v_{\mathbb{R}^{n+1}} Y[\xi] d v+\int_{\Omega}\langle Y[\xi], \bar{N}\rangle d a \\
& =-2(n+1) \int_{T}\langle\xi, x\rangle d v .
\end{aligned}
$$

So generally $\int_{M} \phi d a \neq 0$. That means $\phi[\xi]$ is not a test function.

However, under the hypothesis of Theorem 1 that the mass center of $T$ is at the origin, we have $\int_{M} \phi d a=-2(n+1) \int_{T}\langle\xi, x\rangle d v=0$ for $\forall \xi \in \mathbb{S}^{n}$. So if we choose $\xi$ as an eigenvector corresponding to the negative eigenvalue 
of $Q$, we have $\partial^{2} E(\phi[\xi])=Q(\xi, \xi)<0$, which implies that $M$ is unstable. This completes the proof of Theorem 1 .

\section{Other APPLICATIONS AND QUESTION}

In this section we shall give several applications of the above argument and propose a conjecture on the topic.

4.1. Another criteria for instability. The following proposition is an immediate result.

Proposition 10. If the quadratic form $Q$ has two negative eigenvalues, then $M$ is unstable.

Proof. Assume $Q$ is diagonalized such that $\xi_{1}$ and $\xi_{2}$ are the eigenvectors corresponding to the two negative eigenvalues. Then for real numbers $c_{1}$ and $c_{2}$ with $c_{1}^{2}+c_{2}^{2} \neq 0$,

$$
Q\left(c_{1} \xi_{1}+c_{2} \xi_{2}, c_{1} \xi_{1}+c_{2} \xi_{2}\right)=c_{1}^{2} Q\left(\xi_{1}, \xi_{1}\right)+c_{2}^{2} Q\left(\xi_{2}, \xi_{2}\right)<0 .
$$

On the other hand,

$$
\begin{aligned}
\int_{M} \phi\left[c_{1} \xi_{1}+c_{2} \xi_{2}\right] d a & =-2(n+1) \int_{T}\left\langle c_{1} \xi_{1}+c_{2} \xi_{2}, x\right\rangle d v \\
& =-2(n+1)\left(c_{1} \int_{T}\left\langle\xi_{1}, x\right\rangle d v+c_{2} \int_{T}\left\langle\xi_{2}, x\right\rangle d v\right) .
\end{aligned}
$$

So we can always choose proper $c_{1}$ and $c_{2}$ with $c_{1}^{2}+c_{2}^{2} \neq 0$ such that $\int_{M} \phi\left[c_{1} \xi_{1}+c_{2} \xi_{2}\right] d a=0$. Then using $\phi\left[c_{1} \xi_{1}+c_{2} \xi_{2}\right]$ as a test function, from 23. we know $M$ is unstable.

The significance of the above proposition is as follows. For a given concrete capillary hypersurface $M$ in $B^{n+1}$, the quadratic form $Q$ is computable in principle. Then if $Q$ has two negative eigenvalues, we can assert its instability. Also from this proposition we know that for hyperplanes and spherical caps $Q$ has exactly one negative eigenvalue.

\subsection{The mass center of minimal submanifolds with free boundary.} By free boundary we mean that $M$ intersects $\partial B^{n+1}$ orthogonally, that is, $\nu=x$ along $\partial M$. By analyzing the vector field $Y[\xi]$, we have the following proposition.

Proposition 11. The mass center of a minimal submanifold $M^{k}$ with free boundary in a Euclidean ball is at the origin.

Proof. Along $M^{k}$ choose the orthonormal basis $\left\{e_{i}, i=1, \cdots, k ; e_{\alpha}, \alpha=\right.$ $k+1, \cdots, n+1\}$ such that $\left\{e_{i}, i=1, \cdots, k\right\} \subset T M$. Then we have

$\operatorname{div}_{M} Y[\xi]^{T}=\operatorname{div}_{M}\left(Y[\xi]-\sum_{\alpha}\left\langle Y[\xi], e_{\alpha}\right\rangle e_{\alpha}\right)=2 k\langle\xi, x\rangle+\langle Y[\xi], k \vec{H}\rangle=2 k\langle\xi, x\rangle$. 
By divergence theorem, we have

$$
2 k \int_{M}\langle\xi, x\rangle d a=\int_{\partial M}\left\langle Y[\xi]^{T}, \nu\right\rangle d s=\int_{\partial M}\langle Y[\xi], x\rangle d s=0,
$$

where we have used the fact $Y[\xi]$ is tangential to $\partial B^{n+1}$.

This proposition shows that minimal submanifolds with free boundary have some symmetry. Comparing with it, we mention two other properties of $M^{k}$ :

(1) The mass center of the boundary $\partial M$ is at the origin (a simple argument).

(2) The volume of $M$ has a lower bound $\left|M^{k}\right| \geq\left|B^{k}\right|$, where $B^{k}$ is a $k$-dimensional unit ball ( $3,7,7,14])$.

4.3. Stable immersed closed CMC hypersurfaces in $\mathbb{R}^{n+1}$. At last we give a new proof of a theorem by Barbosa and do Carmo.

Theorem 12 ( [1]). The only stable immersed closed hypersurface of constant mean curvature in $\mathbb{R}^{n+1}$ is the round sphere.

Proof. By translation, assume the mass center of generalized body $T$ enclosed by $M$ is at the origin. So $\int_{M} \phi[\xi] d a=0$ for all $\xi \in \mathbb{S}^{n}$. If $M$ is the round sphere, we are done. Otherwise $|x| \neq$ const. So by Lemma 9 the quadratic form $Q$ has a negative eigenvalue. Choosing $\xi$ as an eigenvector corresponding to the negative eigenvalue, we have

$$
\partial^{2} E(\phi[\xi])=-\int_{M} L \phi[\xi] \cdot \phi[\xi] d a=Q(\xi, \xi)<0,
$$

which shows that $M$ is unstable.

4.4. An open question. Since all the examples, i.e. the Delaunay type capillary hypersurfaces is known to be stable or unstable, we propose a conjecture as follows.

Conjecture 13. The only stable capillary hypersurface $M^{n}(n \geq 3)$ in a unit Euclidean ball $B^{n+1}$ is a totally geodesic hyperplane or a spherical cap.

There are some remarks on this conjecture.

(1) For $n \geq 2, H=0$ and $\theta=\frac{\pi}{2}, M$ must be totally geodesic [14].

(2) For $n=2$ and $\theta=\frac{\pi}{2}, M$ is a totally geodesic disk, a spherical cap or a surface of genus 1 with embedded boundary having at most two connected components 14.

(3) For $n=2$ and $H=0, \vec{M}$ is a totally geodesic disk or a surface of genus 1 with at most three connected boundary components 13 . 


\section{REFERENCES}

[1] J. L. Barbosa and M. do Carmo, Stability of hypersurfaces with constant mean curvature, Math. Z. 185 (1984), no. 3, 339-353.

[2] J. Bokowski and E. Sperner Jr., Zerlegung konvexer Körper durch minimale Trennflächen, J. Reine Angew. Math. 311/312 (1979), 80-100.

[3] S. Brendle, A sharp bound for the area of minimal surfaces in the unit ball, Geom. Funct. Anal. 22 (2012), no. 3, 621-626.

[4] L. Cao and H. Li, r-minimal submanifolds in space forms, Ann. Global Anal. Geom. 32 (2007), no. 4, 311-341.

[5] R. Finn, Equilibrium Capillary Surfaces, Grundlehren der Mathematischen Wissenschaften, vol. 284, Springer-Verlag, New York, 1986.

[6] R. Finn, Capillary surface interfaces, Notices Amer. Math. Soc. 46 (1999), no. 7, $770-781$.

[7] A. Fraser and R. Schoen, The first Steklov eigenvalue, conformal geometry, and minimal surfaces, Adv. Math. 226 (2011), 4011-4030.

[8] A. Fraser and R. Schoen, Sharp eigenvalue bounds and minimal surfaces in the ball, arXiv: 1209.3789 .

[9] A. Fraser and R. Schoen, Minimal surfaces and eigenvalue problems, arXiv: 1304.0851.

[10] E. Gonzalez, U. Massari, and I. Tamanini, Existence and regularity for the problem of a pendent liquid drop, Pacific J. Math. 88 (1980), no. 2, 399-420.

[11] M. Hutchings, F. Morgan, M. Ritoré, and A. Ros, Proof of the double bubble conjecture, Ann. of Math. (2) 155 (2002), no. 2, 459-489.

[12] P. I. Marinov, Stability analysis of capillary surfaces with planar or spherical boundary in the absence of gravity, Ph.D. thesis, The University of Toledo (Ohio), 2010.

[13] A. Ros and R. Souam, On stability of capillary surfaces in a ball, Pacific J. Math. 178 (1997), no. 2, 345-361.

[14] A. Ros and E. Vergasta, Stability for hypersurfaces of constant mean curvature with free boundary, Geom. Dedicata 56 (1995), no. 1, 19-33.

[15] R. Schoen and S.-T. Yau, Lectures on Differential Geometry, Conf. Proc. Lecture Notes Geom. Topol., vol. 1, International Press, 1994.

[16] J. Simons, Minimal varieties in riemannian manifolds, Ann. of Math. (2) 88 (1968) $62-105$.

Department of mathematical sciences, Tsinghua University, 100084, Beijing, P. R. China

E-mail address: hli@math.tsinghua.edu.cn

Department of mathematical sciences, Tsinghua University, 100084, BeiJing, P. R. CHINA

E-mail address: xiongcw10@mails.tsinghua.edu.cn 